\title{
Subsurface Resistivity Measurements Using Square Waveforms
}

\author{
Manel Gasulla-Forner, Student Member, IEEE, Josep Jordana-Barnils, Student Member, IEEE, \\ Ramón Pallás-Areny, Fellow, IEEE, and Josep Maria Torrents, Member, IEEE
}

\begin{abstract}
This work analyzes the effect of inductive and capacitive coupling between the injecting circuit and the detecting circuit in resistive field surveys. Theoretical and experimental results demonstrate that if a square waveform is injected into the soil, and synchronous sampling is used to sample at the flat zone of the detected voltage, then the effect of the interference is greatly reduced. Furthermore, square waveforms are easier to generate than sinusoidal waveforms, so they offer a new approach to subsurface resistivity measurements.
\end{abstract}

Index Terms - Conductivity measurement, data acquisition, electromagnetic coupling, sampling methods, soil measurements, synchronous detection.

\section{INTRODUCTION}

$\mathbf{T}$ HE detection of buried structures from the surface without drilling into the soil is of interest in archaeology and in other applications such as detection of water and contaminants from leaking underground pipes. Ground penetrating radar and time domain reflectometry are techniques that can be applied in these cases. Our work is concerned with geoelectrical prospecting methods, which consist of injecting a current to the soil with a pair of electrodes and detecting the drop in voltage with another pair of electrodes.

The parameter that provides information on the buried structure (the anomaly) is the apparent resistivity $\rho_{a}$, which is given by $\rho_{a}=k \Delta V / I$ [1]. In this expression $\Delta V$ is the detected voltage, $I$ is the injected current, and $k$ is a geometric factor, which depends on the configuration of the electrode array. It is important to recognize that any possible error in the measured voltage will affect $\rho_{a}$ and can hinder the detection of the anomaly. Some error sources are the position of the electrodes, telluric noise (which has its main influence in dc measurements) and electromagnetic coupling between the injecting and detecting circuits in ac measurements.

In electrical surveys, electromagnetic induction between current and voltage cables must be avoided. This is easily achieved by using dipole-dipole arrays [2], but it is interesting to devise a measurement method able to reduce this interference independently of electrode configuration.

\section{Problem Statement}

In electrical impedance measurement, we inject a current (frequency $f_{s}$ ) and detect a drop in voltage whose amplitude

Manuscript received June 1, 1997; revised April 1, 1998. This work was supported by the Spanish DGICYT under Project PB93-0961.

The authors are with the Divisió d'Instrumentació i Bioenginyeria, Departament d'Enginyeria Electrònica, Universitat Politècnica de Catalunya, 08034 Barcelona, Spain.

Publisher Item Identifier S 0018-9456(98)05483-7.

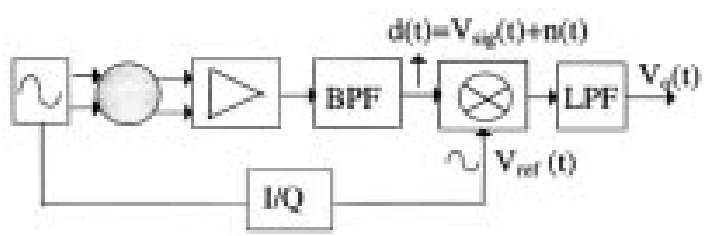

Fig. 1. Impedance measurement by homodyne detection.

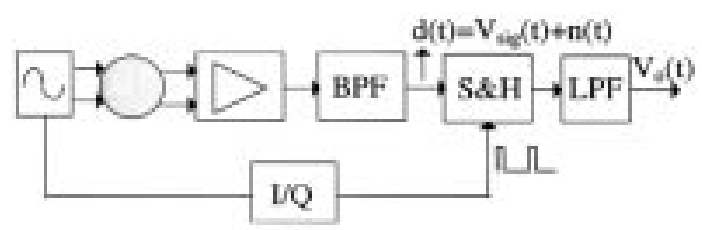

Fig. 2. Impedance measurement by synchronous sampling.

is modulated by the impedance sensed. Modulation produces an upward translation of the information spectrum. Demodulation, therefore, implies a downward frequency translation in order to recover the information from the modulated wave.

A common demodulation technique providing a good signal-to-noise ratio is homodyne detection. This method can be applied to impedance measurement as shown in Fig. 1.

If we assume that the signal detected is $V_{\text {sig }}(t)=$ $\sqrt{2} V_{s} \cos \left(2 \pi f_{s} t+\phi_{s}\right)$ and the reference signal is $V_{r e f}(t)=$ $\sqrt{2} V_{r} \cos 2 \pi f_{r} t$, then the demodulated signal at the output of the low pass filter, when $f_{s}=f_{r}$, is $V_{o}(t)=V_{s} V_{r} \cos \phi_{s}$. In a resistive medium, $\phi_{s}=0$, then $V_{o}(t)=V_{s} V_{r}$.

If there is an interfering signal $n(t)=\sqrt{2} V_{i} \cos \left(2 \pi f_{i} t+\right.$ $\phi_{i}$ ), having the same frequency than the carrier, typically capacitively or inductively coupled, then the demodulated signal in a resistive medium is $V_{o}(t)=V_{r}\left(V_{s}+V_{i} \cos \phi_{i}\right)$. If the phase angle $\phi_{i}$ of the interference is $90^{\circ}$ then the signal will be recovered without any error. But when $\phi_{i} \neq 90^{\circ}$ there will be a measurement error. In principle, the larger the frequency of the injected current, the larger the interference will be, because both inductive and capacitive interference increase with frequency. In extreme cases the detector can even saturate because of the interference.

Impedance measurement by synchronous sampling (Fig. 2) is another phase-sensitive amplitude demodulation technique [3]. If $d(t)$ is sampled at $t=n T, n$ integer, $T$ being the period of the signal, that is, if the signal $V_{s i g}(t)$ is sampled at its maximal value, then the detected signal will be $V_{o}(t)=\sqrt{2} V_{s}\left(1+\left(V_{i} / V_{s}\right) \cos \phi_{i}\right)$. It can be seen that the measurement error also depends on the phase angle $\phi_{i}$ of 


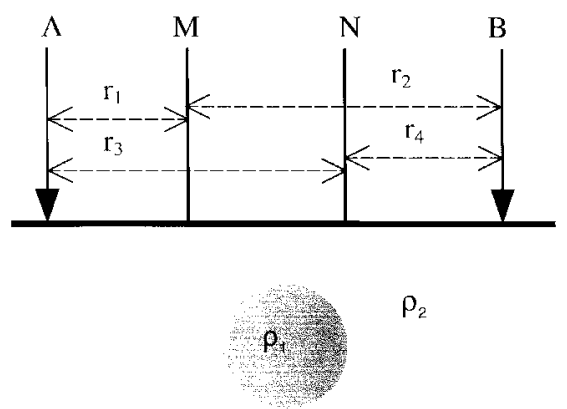

Fig. 3. Linear array of current and potential electrodes.

the interference. But here this drawback can be avoided by injecting a square waveform to the soil instead of a sinusoidal waveform [4]. In this case the detected signal is sampled at a zero-slope point, thus avoiding the stray current coupling from the injecting circuit to the measuring circuit ("transformer effect"). Therefore, this allows us to measure at a frequency high enough in order for electrode impedance to be relatively low.

Electromagnetic interference is particularly troublesome in subsurface resistivity measurements because of the physical dimensions and arrangement of circuits. Fig. 3 shows the principle of this technique [1]. A current of strength $I$ is injected by electrodes $A$ and $B$. The potential difference between points $M$ and $N$ in a homogeneous medium is

$$
\Delta V=V_{M}-V_{N}=\frac{I \rho}{2 \pi}\left[\left(\frac{1}{r_{1}}-\frac{1}{r_{2}}\right)-\left(\frac{1}{r_{3}}-\frac{1}{r_{4}}\right)\right]
$$

where $\rho$ is the soil resistivity, and $r_{1}$ to $r_{4}$ are distances between the electrodes. The apparent resistivity $\rho_{a}$ is obtained by solving (1) for $\rho$ :

$$
\rho_{a}=k \frac{\Delta V}{I}
$$

where $k$ is the geometric factor.

The apparent resistivity provides information about the presence of an anomaly. The potential difference $\Delta V$ can be affected by the electromagnetic coupling from the injecting circuit to the detecting circuit, which will alter the value of $\rho_{a}$.

There are two main coupling mechanisms: capacitive coupling and inductive coupling [5]. Capacitive coupling arises from the parasitic capacitances between injecting and detecting wires. Inductive coupling appears because the injecting and detecting circuits behave like the primary and secondary winding of an ordinary transformer.

If $\mathrm{dc}$ currents are used, then there is neither capacitive nor inductive coupling. But dc currents pose another important problem: polarization potentials generated at the contact between a metallic conductor (the electrode) and an electrolytic conductor (the moist ground). These polarization potentials are $\mathrm{dc}$ voltages that mask the measured voltage difference.

Interference by capacitive and inductive coupling results only when there is a change in the voltage or current in the injecting circuit. For a sinusoidal signal, this means the entire waveform except at its maximum and minimum. For a square signal, however, interference will result only during transition

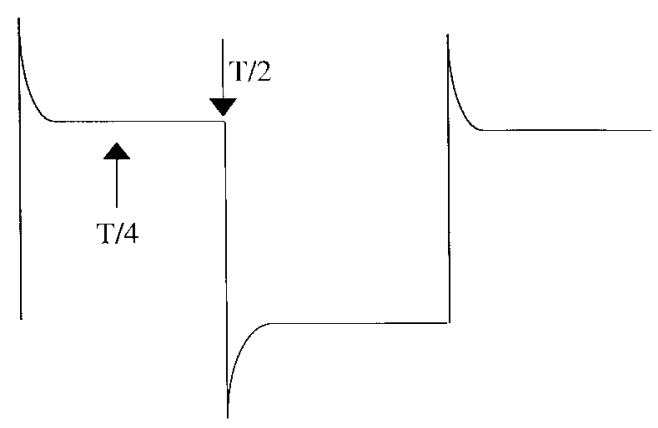

Fig. 4. Synchronous sampling with square waveforms. From $T / 4$ to $T / 2$ the interference is minimal.

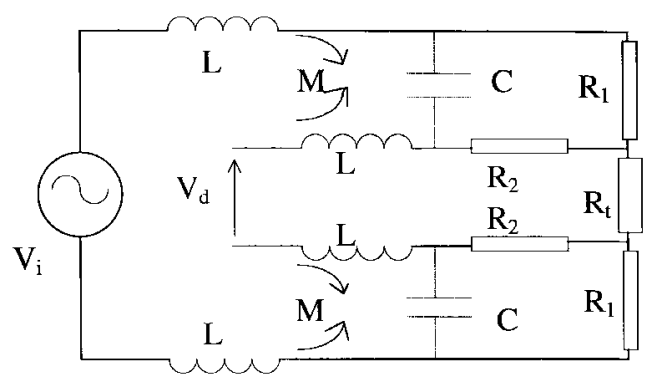

Fig. 5. Inductive and capacitive coupling between injecting and detecting circuits.

times. Hence, by sampling at $T / 4$ or a bit later the effect of the interference should disappear (Fig. 4).

\section{THEORETICAL ANALYSIS}

Fig. 5 shows the circuit model of a resistivity measurement. $V_{i}$ and $V_{d}$ are the injected and detected voltages, respectively, $C$ is the capacitance between the injecting and detecting wires, $L$ and $M$ are the self and mutual inductance of cables, $R_{1}$ and $R_{2}$ are the contact resistances of the injecting and detecting electrodes, and $R_{t}$ is the resistance between the electrodes $M$ and $N$. For simplicity, we study capacitive and inductive interferences separately.

If no interference is present, the ideal detected voltage will be

$$
V_{d o}=\frac{R_{t}}{R_{t}+2 R_{1}} \cdot V_{i}
$$

We define the relative error in the measured voltage as

$$
e_{r}=\frac{V_{d}-V_{d o}}{V_{d o}}
$$

where $V_{d}$ is the detected voltage contaminated by the interference. We consider an injected frequency of up to $10 \mathrm{kHz}, R_{t}$ about $0.33 \Omega, R_{1}$ and $R_{2}$ in the range of $50 \Omega$ to $5 \mathrm{k} \Omega, C$ ranging from $10-100 \mathrm{pF}$, and $L$ and $M$ between 10 and 100 $\mu \mathrm{H}$. Either for sinusoidal and square waveforms we sample $V_{d}$ at $T / 4+n T$, where $T$ is the period of the signal and $n$ is an integer.

First of all, we consider the effect of stray capacitances. When we inject a square voltage whose peak value is $V_{i p}$ the 
detected voltage is

$$
V_{d} \approx V_{i p}\left(\frac{R_{t}}{R_{t}+2 R_{1}}+\frac{4 R_{1}}{R_{t}+2 R_{1}} e^{-t / C R_{2}}\right)
$$

and the relative error for the assumed value range for the different parameters is

$$
e_{r} \approx \frac{4 R_{1}}{R_{t}} e^{-t / C R_{2}}
$$

If the generator injects a sinusoidal waveform, the relative error in the measured voltage for the in phase component is

$$
e_{r} \approx \frac{2 \omega^{2} C^{2} R_{2}^{2} R_{1}}{R_{t}}
$$

It can be seen that the error greatly increases as $R_{2}$ and $C$ become larger. For example, if the frequency is $10 \mathrm{kHz}$, $C=10 \mathrm{pF}$, and $R_{1}=R_{2}=500 \Omega$ the relative error for sinusoidal signals is only $3 \times 10^{-4}$ but when $C=100 \mathrm{pF}$ and $R_{1}=R_{2}=5 \mathrm{k} \Omega$ the relative error increases to 30 . If square waveforms are used the error is negligible in both cases.

Secondly, we consider the effect of inductive coupling. The relative error for square signals in the same conditions as above is

$$
e_{r} \approx \frac{4 R_{1}}{R_{t}} e^{\left(-R_{1} / L\right) t}
$$

and for sinusoidal signals is

$$
e_{r} \approx \frac{2 \omega^{2} M L}{R_{t} R_{1}}
$$

It is interesting to point out that if $R_{1}$ is reduced the error increases in both cases. For sinusoidal signals the relative error varies from $48 \times 10^{-4}$ when $L=M=10 \mu \mathrm{H}$ and $R_{1}=R_{2}=500 \Omega$, to 4.68 when $L=M=100 \mu \mathrm{H}$ and $R_{1}=R_{2}=50 \Omega$. This error is minimized to $22 \times 10^{-4}$ when using square waveforms.

Electromagnetic coupling increases with frequency. To avoid the effect of interference in the measured voltage it is advisable therefore to inject a square waveform into the soil instead of a sine wave and to use a detector based on synchronous sampling.

\section{Materials AND MethodS}

In order to quantify interference in a field survey, we carried out some measurements in a typical soil of our campus. Fig. 6 shows the electrode arrangement. The injecting and detecting cables were arranged in a triangular geometry parallel to the ground surface. The transmitter and the receiver were placed in a vertex (TD) of this triangle, in front of the electrode array. The generator was an HP3245A and the detector was a fully differential synchronous demodulator, based on synchronous sampling [6]. The four electrodes were made from stainless steel, $20 \mathrm{~cm}$ in length and $1 \mathrm{~cm}$ in diameter. They were inserted several centimeters into the ground to ensure a good electrical contact. Connecting wires were $1 \mathrm{~mm}$ in diameter and had

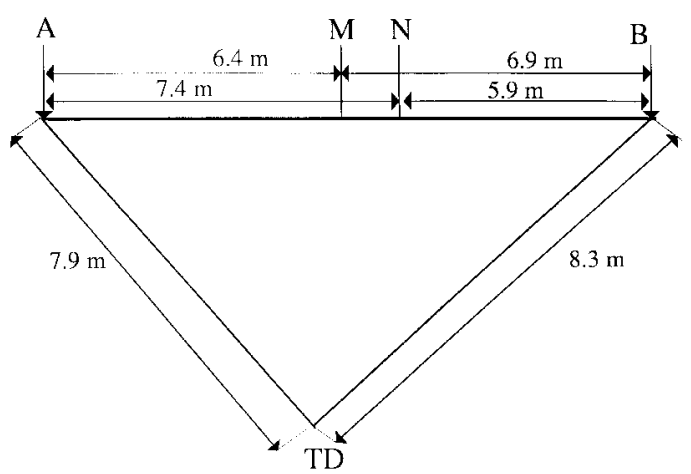

Fig. 6. Field survey: electrode array and wire arrangement.

plastic insulation, which avoided any possible direct leakage current to the ground.

The HP3245 generates the sinusoidal/square signal of 20 $\mathrm{V}_{\mathrm{pp}}$ and the signal reference for the sampler. The sampling frequency was the same that the input signal $\left(f_{r}=f_{s}\right)$ and its values were $100 \mathrm{~Hz}, 1 \mathrm{kHz}$, and $10 \mathrm{kHz}$. The detector had a gain of 100 . The duty cycle for the sampling signal was $10 \%$. The sampling instant was at $T / 4+n T$. The drop in voltage monitored the injected current across a $10 \Omega$ resistor in series with an injecting wire, by means of a portable oscilloscope (Tektronix THS-710E).

\section{EXPERIMENTAL RESUlTS}

In order to quantify electromagnetic interference, we measured first the capacitive coupling, secondly the inductive interference and finally their combined effect in the differential voltage measured between electrodes $M$ and $N$.

Capacitive interference increases in value if coupled to a high-impedance circuit. In order to demonstrate this effect, a variable resistor was connected to the end of two twisted cables. The detected potential increased with the resistor value. By placing a resistor of $5 \mathrm{k} \Omega$, at $10 \mathrm{kHz}$ and by sampling at $T / 4$ of the injected signal, $141 \mathrm{mV}$ were detected at the output for a sinusoidal waveform and only $14 \mathrm{mV}$ for a square waveform. Capacitive coupling increased with frequency and with cable length. Inductive interference was minimal because the detecting wires were twisted.

If the area of the detecting circuit is increased and its terminals short-circuited, capacitive interference is negligible (zero dependence) and inductive coupling predominates. At $10 \mathrm{kHz}$ the detector output was $23 \mathrm{mV}$ when using sinusoidal signals and only $2 \mathrm{mV}$ by injecting square waveforms. Inductive coupling increased with the area of the detecting wires.

Table I shows the demodulated dc voltage $V_{o}$ (once multiplied by 100) and the injected current (peak to peak) with sinusoidal and square waveforms at frequencies 1 and $10 \mathrm{kHz}$. The detector was connected to electrodes $M$ and $N$. In this case there was, added to the ideal voltage, both capacitive and inductive interference and they increased with frequency. However, when using square waveforms the detected voltage was similar, which confirms our predictions that sampling in the flat zone of the square waveform, minimizes the effect of interference. When using sinusoidal waveforms, the voltage 
TABLE I

Detected Voltage when InTerference Coupling is MaXimum

\begin{tabular}{l|l|l}
\hline Injected wave & $1 \mathrm{kHz}$ & $10 \mathrm{kHz}$ \\
\hline $\begin{array}{l}\text { Sinusoidal } \\
\text { Sampling } \\
\text { instant=T/4 }\end{array}$ & $\mathrm{V}_{0}=337 \mathrm{mV}$ & $\mathrm{V}_{0}=644 \mathrm{mV}$ \\
& & \\
\hline $\begin{array}{l}\text { Square } \\
\text { Sampling } \\
\text { instant=T/4 }\end{array}$ & $\mathrm{V}_{0}=351 \mathrm{mV}$ & $\mathrm{V}_{0}=370 \mathrm{mV}$ \\
& $\mathrm{I}=19 \mathrm{~mA}$ & $\mathrm{I}=21 \mathrm{~mA}$ \\
\hline
\end{tabular}

TABLE II

Detected Voltage when Detecting Cables are Twisted

\begin{tabular}{l|l|l}
\hline \multicolumn{1}{c|}{$\begin{array}{c}\text { Injected } \\
\text { waveform }\end{array}$} & $1 \mathrm{kHz}$ & $10 \mathrm{kHz}$ \\
\hline $\begin{array}{l}\text { Siusoidal } \\
\text { Sampling } \\
\text { instant=T/4 }\end{array}$ & $\begin{array}{l}\mathrm{V}_{0}=349 \mathrm{mV} \\
\mathrm{I}=19.4 \mathrm{~mA}\end{array}$ & $\begin{array}{l}\mathrm{V}_{0}=437 \mathrm{mV} \\
\mathrm{I}=20.8 \mathrm{~mA}\end{array}$ \\
$\begin{array}{l}\text { Square } \\
\begin{array}{l}\text { Sampling } \\
\text { instant=T/4 }\end{array}\end{array}$ & $\begin{array}{l}\mathrm{V}_{0}=356 \mathrm{mV} \\
\mathrm{I}=19.4 \mathrm{~mA}\end{array}$ & $\begin{array}{l}\mathrm{V}_{\mathrm{o}}=371 \mathrm{mV} \\
\mathrm{I}=21.2 \mathrm{~mA}\end{array}$ \\
\hline
\end{tabular}

detected changed by $91 \%$. The small change observed in the square wave is principally due to the decreasing value in the contact resistance $R_{1}$ with frequency, which increases the injected current.

If the detecting cables were twisted, interference reduced because the distance between detecting wires and injecting wires increases and the area of the detecting circuit was much smaller. The results are shown in Table II.

\section{CONCLUSIONS}

We have proposed a simple model to study the effects of capacitive and inductive interference in subsurface resistivity measurements. Theoretical analysis shows that injecting a square waveform into the soil instead of a sine wave and using synchronous sampling diminishes the effect of the interference.

Experimental results in a typical soil of our campus show that capacitive interference increases with the electrode impedance, cable length and frequency. Then watering the electrodes reduces the capacitive interference. Inductive coupling increased with the area of the detecting wires and with the frequency. Twisting the detecting cables reduces the interference. However, in any case the interference effect is much smaller when using square waveforms. This is because synchronous sampling allows taking samples in the interval $T / 4-T / 2$, when interference effect has disappeared.

\section{REFERENCES}

[1] W. M. Telford, L. P. Geldart, and R. E. Sheriff, Applied Geophysics, 2nd ed. Cambridge, U.K.: Cambridge Univ. Press, 1990.

[2] J. Milsom, Field Geophysics. New York: Open Univ. Press and Halsted, 1988.

[3] R. Pallás-Areny and O. Casas, "A novel synchronous demodulator for ac signals," IEEE Trans. Instrum. Meas., vol. 45, pp. 413-416, Apr. 1996.

[4] R. Pallás-Areny and J. A. Brescolí, "Electrical impedance measurements by synchronous sampling applied to square waveforms," in IEE Colloq. Innovations in INstrumentation for Electrical Tomography Dig., 1995/099.

[5] O. Koefoed, Geosounding Principles, 1-Resistivity Sounding Measurements. Amsterdam, The Netherlands: Elsevier, 1988.

[6] M. Gasulla, J. Jordana, R. Pallás-Areny, and J. M. Torrents, "The floating capacitor as a differential building block," in IEEE Instrumentation and Measurement, Technology Conf., Ottawa, Ont., Canada, May 19-21, 1997.

Manel Gasulla-Forner (S'97), for a photograph and biography, see this issue p. 29.

Josep Jordana-Barnils (S'93), for a photograph and biography, see this issue p. 29.

Ramón Pallás-Areny (F'98), for a photograph and biography, see this issue p. 29.

Josep Maria Torrents (M'90), for a photograph and biography, see this issue p. 29. 